\title{
ESTADO TRÓFICO DOS ESTUÁRIOS DOS RIOS IPOJUCA E MEREPE - PE
}

\author{
Thayse Natália Freitas BATISTA ${ }^{1}$ \\ Manuel de Jesus FLORES MONTES ${ }^{1,2}$
}

Recebido em: julho/2013

Aceito em: agosto/2013

\section{RESUMO}

Este estudo objetivou avaliar o estado trófico e a qualidade das águas estuarinas, dos rios Ipojuca e Merepe (PE), através do índice TRIX. Foram realizadas 7 coletas de água, em 3 pontos, na superfície, em diferentes estágios de maré de sizígia, com a garrafa Niskin, durante um ciclo sazonal (de maio/09 a maio/10), com freqüência bimestral. A distribuição sazonal do TRIX nestes estuários esteve mais elevada no período chuvoso em ambos os estágios de maré. Os valores do TRIX variaram de 5,41 a 7,83 , onde os menores índices foram registrados no $\mathrm{P} 1$ durante a preamar, no período chuvoso, e os maiores índices foram encontrados no P3 durante a baixa-mar, no mesmo período. Estados tróficos variando de alto a elevado, atestam a má qualidade destas águas e traduzem a forte pressão antrópica sobre estes ecossistemas.

Palavras chave: eutrofização, TRIX, áreas estuarinas.

\section{ABSTRACT}

This study aimed to evaluate the trophic status and the quality of estuarine waters from the rivers Ipojuca and Merepe (PE) throughout the TRIX index. Seven water sampling were carried out at three points on the surface at different stages of spring tide, with the Niskin bottle during a seasonal cycle (from May/09 to May/10), with a bimonthly frequency. The TRIX seasonal distribution in these estuaries was higher in the rainy season in both tidal stages. The TRIX values ranged from 5.41 to 7.83 , where the lowest rates were registered in $\mathrm{P} 1$ during high tide in the rainy season, and the highest rates were found in P3 during low tide, in the same period. Trophic states ranging from high to elevated attest to the poor quality of these waters and reflect the strong anthropic pressure on these ecosystems.

Key words: eutrophication, TRIX, estuarine areas.

\section{INTRODUÇÃO}

Nas últimas décadas, vem sendo registrado o aumento do lançamento de efluentes nos cursos d'água, em virtude do crescimento populacional e econômico. Estes efluentes elevam a carga orgânica e o teor de nutrientes dos ecossistemas aquáticos, e resultam em uma série de impactos ambientais. Tais nutrientes são despejados de forma dissolvida ou particulada em lagos, represas e rios, sendo transformados em partículas orgânicas, matéria viva vegetal, pelo metabolismo das plantas e carreados para região costeira (TUNDISI, 2003).

Um dos principais problemas acarretado pelo aumento da quantidade de matéria orgânica disponível na massa de água é o processo de eutrofização (NIXON, 1995). Sendo este um processo natural de adição de nutrientes, sobretudo nitrogênio $(N)$ e fósforo $(P)$, em águas costeiras provenientes das bacias de drenagens. Entretanto as atividades humanas têm acelerado este processo (FERREIRA et al., 2007), que eleva a produção primária no sistema causando florações de algas, reduzindo a transparência da água e resultando em condições de hipoxia e anoxia, provocando danos a biodiversidade marinha com prejuízos sociais e econômicos (VASCETTA et al., 2004). 
Segundo, Cloern (2001), um grande número de parâmetros de causa e efeito são envolvidos no processo de eutrofização, influenciando negativamente a saúde dos sistemas costeiros. Neste sentido, a utilização de índices multivariados que simplificam uma série de variáveis bióticas e abióticas em valores inteiros, de fácil compreensão pelo público, torna-se uma ferramenta cada vez mais utilizada no gerenciamento ambiental (SALAS et al., 2008). A necessidade de avaliar os processos eutróficos em regiões estuarinas e costeiras, a fim de dar apoio a decisões políticas tem levado ao desenvolvimento de diferentes métodos os quais usam avaliações baseadas em sistemas multiparâmetros (BRICKER et al., 2003; VOLLENWEIDER et al., 1998). Um destes índices é o TRIX (Trophic Index), desenvolvido por Vollenweider et al. (op cit.), que utiliza variáveis ambientais diretamente medidas e coletadas rotineiramente, como concentração de clorofila $a$, saturação de oxigênio dissolvido, concentração de nitrogênio inorgânico dissolvido e fósforo total, para identificar em qual estágio do processo de eutrofização o estuário se encontra.

Estudos relativos à eutrofização estuarina utilizando a aplicação de índices são bastante escassos no nordeste brasileiro, em especial no estado de Pernambuco. Neste sentido, 0 presente estudo teve por objetivo avaliar o estado trófico e a qualidade das águas estuarinas, dos rios Ipojuca e Merepe, exportadas para a área costeira utilizando o índice TRIX, a fim de formar um banco de dados que sirva de referência para futuros monitoramentos ambientais.

\section{ÁREA DE ESTUDO}

O estuário do rio Ipojuca está localizado no município de Ipojuca $\left(08^{\circ} 15^{\prime} 00^{\prime \prime}\right.$ e $08^{\circ} 30^{\prime} 00^{\prime \prime}$ $\mathrm{S}, 34{ }^{\circ} 55^{\prime} 00^{\prime \prime}$ e $35^{\circ} 05^{\prime} 00^{\prime \prime} \mathrm{W}$ ) a cerca de $40 \mathrm{~km}$ ao sul da cidade do Recife, Pernambuco (Figura 1). O clima, segundo Köppen, é do tipo As', tropical - quente e úmido, com chuvas de outonoinverno. O regime pluviométrico varia entre 1.850 a $2.364 \mathrm{~mm}$ anuais. A temperatura média anual é de $24^{\circ} \mathrm{C}$, umidade relativa média anual superior a $80 \%$, com predominância dos ventos de sudeste (NIMER, 1979).

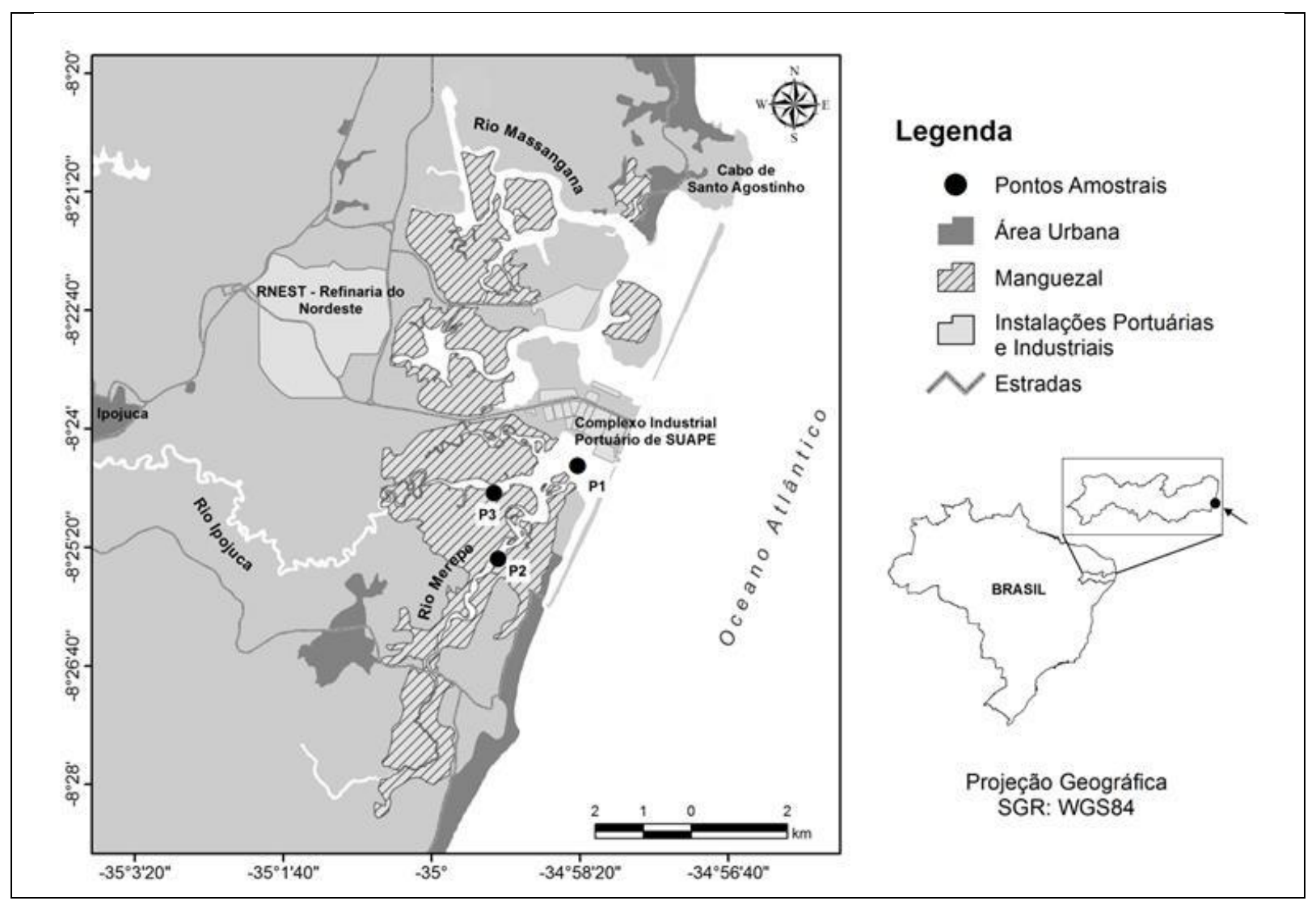

Figura 1 - Localização geográfica dos pontos de coleta (P1: Confluência dos rios Ipojuca e Merepe; P2: rio Merepe e P3: rio Ipojuca). 
O rio Ipojuca nasce no Agreste pernambucano, percorre aproximadamente $250 \mathrm{~km}$, destes $15 \mathrm{~km}$ são de área estuarina, até desaguar no Oceano Atlântico. Ao longo de seu percurso drena uma área de $3.514,35 \mathrm{Km}^{2}$, passando por 25 municípios e atendendo a uma população de aproximadamente 568.630 pessoas (CONDEPE, 1983; $\mathrm{CPRH}, 2003$ ).

Os usos múltiplos de suas águas vão desde abastecimento público a recepção de efluentes. Já o uso do solo ao longo da bacia destina-se a ocupação urbana e industrial, áreas cultivadas com cana-de-açúcar, policulturas, pecuária e áreas com remanescentes de Mata Atlântica e Manguezal. Esses múltiplos usos das águas e do solo do rio Ipojuca ao longo de seu percurso, faz com que este receba uma carga orgânica poluidora diária muito elevada (CPRH, op cit.).

O rio Merepe é um rio litorâneo, apresenta um extenso manguezal, próximo a sua foz encontra-se com o rio Ipojuca, desaguando juntos ao sul do Porto de Suape. Embora seja de dimensão reduzida, desempenha um importante papel na manutenção dos ecossistemas e das comunidades em sua área de influência ( $\mathrm{CPRH}$, op cit.).

A instalação do Complexo Industrial Portuário de Suape (CIPS), em uma área de grande importância ecológica, causou uma série de impactos ambientais, como a alteração das características dos ecossistemas estuarinos e deterioração da qualidade de suas águas. Dentre estes estuários, os dos rios Ipojuca e Merepe, que já sofriam com o lançamento de dejetos orgânicos, agrícolas e industriais ao longo de seus cursos d'água, tiveram seus problemas agravados com a supressão de extensas áreas de manguezal, o aterro da foz desses rios, a construção de uma desembocadura artificial e o bloqueio da comunicação desses rios com o mar, devido à construção de molhes para atracagem de navios. Todas essas alterações resultaram no represamento do fluxo de suas águas, ocasionando inundações nas áreas utilizadas pela agroindústria da cana-de-açúcar (CONDEPE, op cit; BRAGA et al., 1989; CPRH, op cit.).

$\mathrm{Na}$ tentativa de minimizar esses impactos, foi feita uma abertura no próprio recife, próxima à desembocadura do rio Ipojuca, para permitir a conexão deste com o mar. Como consequência, constatou-se um retardo de mais de duas horas na maré dinâmica, registrandose atualmente 8 horas de baixa-mar e apenas 4 horas de preamar ao invés dos ciclos normais de 6 horas. As marés apresentam amplitude máxima em torno de 2 metros na desembocadura. As modificações provocaram ainda um forte assoreamento e grande deposição de sedimentos em suspensão na área estuarina, aumentando a turbidez da água e transformando a foz do estuário do rio Ipojuca numa laguna costeira que, em conseqüência da diminuição da profundidade local, causou o aumento da salinidade (NEUMANN, 1991; NEUMANN et al., 1998).

\section{MATERIAL E MÉTODOS}

Durante o período de maio de 2009 a maio de 2010, foram realizadas 07 (sete) campanhas, com freqüência bimestral, completando um ciclo sazonal, onde os meses de (maio/09, julho/09, março/10 e maio/10) correspondem ao período chuvoso, e (setembro/09, novembro/09 e janeiro/10) correspondem ao período de estiagem.

As amostragens foram efetuadas na camada superficial, durante os estofos de baixa-mar $(B M)$ e preamar (PM), nas marés de sizígia, com auxílio da garrafa oceanográfica de Niskin. Em três pontos fixos de coleta (variação espacial) P1, P2 e P3, conforme apresentado na figura 1. Estes pontos foram georreferenciados com o auxílio de um GPS (Sistema de Posicionamento Global) Garmin, levando-se em consideração as características gerais da área de estudo.

As amostras hidrológicas foram processadas e analisadas nos laboratórios de Oceanografia Química e de Produtividade Primária do Departamento de Oceanografia da Universidade Federal de Pernambuco (UFPE). A tabela 1 apresenta os parâmetros analisados e as respectivas técnicas analíticas utilizadas para a sua quantificação.

Tabela 1 - Parâmetros analisados e métodos analíticos utilizados.

PARÂMETRO MÉTODO ANALÍtICO REFERÊNCIA




\begin{tabular}{|c|c|c|}
\hline Temperatura $\left(\mathrm{T}-{ }^{\circ} \mathrm{C}\right)$ & $\begin{array}{l}\text { Termômetro oceanográfico de } \\
\text { inversão, acoplado à garrafa } \\
\text { de coleta, com precisão de } \\
\pm 0,02{ }^{\circ} \mathrm{C} \text {. }\end{array}$ & \\
\hline Salinidade & Mohr-Knudsen & Strickland; Parsons, 1972 \\
\hline Oxigênio Dissolvido (OD - $\mathrm{ml}^{.} \mathrm{L}^{-1}$ ) & Winkler, modificado & Strickland; Parsons, 1972 \\
\hline $\begin{array}{l}\text { Percentual de saturação do } \\
\text { oxigênio dissolvido (OD\%) }\end{array}$ & $\begin{array}{l}\text { Calculado através da } \\
\text { correlação com os valores de } \\
\text { temperatura e salinidade, de } \\
\text { acordo com a tabela-padrão } \\
\text { da "International } \\
\text { Oceanographic Tables" }\end{array}$ & UNESCO, 1973 \\
\hline $\begin{array}{l}\text { Nitrogênio inorgânico dissolvido } \\
\left(\text { NID }-\mu \mathrm{g} \mathrm{I}^{-1}\right)\end{array}$ & Espectrofotométrico & Grasshoff et al.,1983 \\
\hline Fósforo Total (PT - $\mu \mathrm{g} . \mathrm{L}^{-1}$ ) & Espectrofotométrico & Grasshoff et al.,1983 \\
\hline Clorofila a $\left(\mathrm{Cl} a-\mu \mathrm{g} \cdot \mathrm{L}^{-1}\right)$ & Espectrofotométrico & $\begin{array}{l}\text { UNESCO, 1966, segundo } \\
\text { Parsons; Strickland, } 1963\end{array}$ \\
\hline
\end{tabular}

A profundidade local $(\mathrm{m})$ foi determinada in situ com o auxílio de uma ecossonda manual digital LCD Sounder Plastimo Echotest (714700). A transparência da água $(\mathrm{m})$ foi medida determinando-se a profundidade do desaparecimento do disco de Secchi. A altura das marés $(\mathrm{m})$, referente ao período de coleta, foi obtida através da Tábua de Marés editada pela Diretoria de Hidrografia e Navegação (DHN) da Marinha do Brasil, tendo-se como referência o Porto de Suape. Os dados pluviométricos $(\mathrm{mm})$ foram fornecidos pelo banco de dados do Laboratório de Meteorologia de Pernambuco (LAMEPE), vinculado ao Instituto de Tecnologia de Pernambuco (ITEP).

Para a caracterização do estado trófico dos sistemas foi utilizado o índice multivariado TRIX (VOLLENWEIDER et al., 1998), o qual utiliza os parâmetros: clorofila a (Cl $\left.a-\mu g . \mathrm{L}^{-1}\right)$, saturação de oxigênio dissolvido (OD\%), nitrogênio inorgânico dissolvido (NID - $\mu g . L^{-1}$ ) e fósforo total (reativo solúvel) (PT - $\mu \mathrm{g} \cdot \mathrm{L}^{-1}$ ), para quantificar a eutrofização estuarina (Rodriguez, 2008). Onde os dois primeiros refletem diretamente a produtividade (estado), enquanto os dois últimos são fatores nutricionais (pressão). Assim, o TRIX é calculado conforme a equação a seguir:

$$
\operatorname{TRIX}=\frac{\left(\log _{10}^{(C l a * \mathrm{OD} \% * \mathrm{NID} * \mathrm{PT})}+K\right)}{m}
$$

As constantes $\mathrm{k}=1,5$ e $\mathrm{m}=1,2$, são valores de escalas introduzidos para ajustar o valor limite mais baixo do índice e a extensão da escala trófica relacionada, de 0 a 10 unidades TRIX, onde serão utilizados os valores expressos na tabela 2 para descrever os sistemas

Tabela 2 - Classificação do Estado Trófico para águas estuarinas de acordo com o TRIX (VOLLENWEIDER et al., op cit. )

\begin{tabular}{ccc}
\hline Escala TRIX & $\begin{array}{c}\text { Estado da qualidade } \\
\text { da água }\end{array}$ & \multicolumn{1}{c}{$\begin{array}{c}\text { Características da } \\
\text { água }\end{array}$} \\
\hline $0-4$ & Alta & Nível trófico baixo \\
$4-5$ & Boa & Nível trófico médio \\
$5-6$ & Mal & Nível trófico alto \\
$6-10$ & Pobre & Nível trófico elevado \\
\hline
\end{tabular}

\section{RESULTADOS}

A aplicação do índice TRIX, segundo a classificação de Vollenweider et al.(1998), nas águas estuarinas dos rios Ipojuca e Merepe indicou que as mesmas se encontram num estado trófico de alto a elevado, sofrendo um forte processo de eutrofização, como mostra a figura 2. 
Os valores de TRIX obtidos no ponto (P1) variaram de 5,41 a 7,50 correspondendo a níveis tróficos de alto a elevado e águas de má qualidade (Tabela 2), onde os menores índices foram registrados durante a preamar, no período chuvoso, com mediana 6,15 . O ponto (P2) apresentou valores elevados do TRIX ao longo de todo período amostral, variando entre 6,07 e 7,50, correspondendo a águas de baixa qualidade. Enquanto que o ponto (P3) apresentou valores de TRIX variando de 5,63 a 7,83, correspondendo a níveis tróficos de alto a elevado, onde os maiores índices foram encontrados na baixa-mar, no período chuvoso, com mediana 7,31, apresentando-se esta como a estação mais críticapara o sistema.

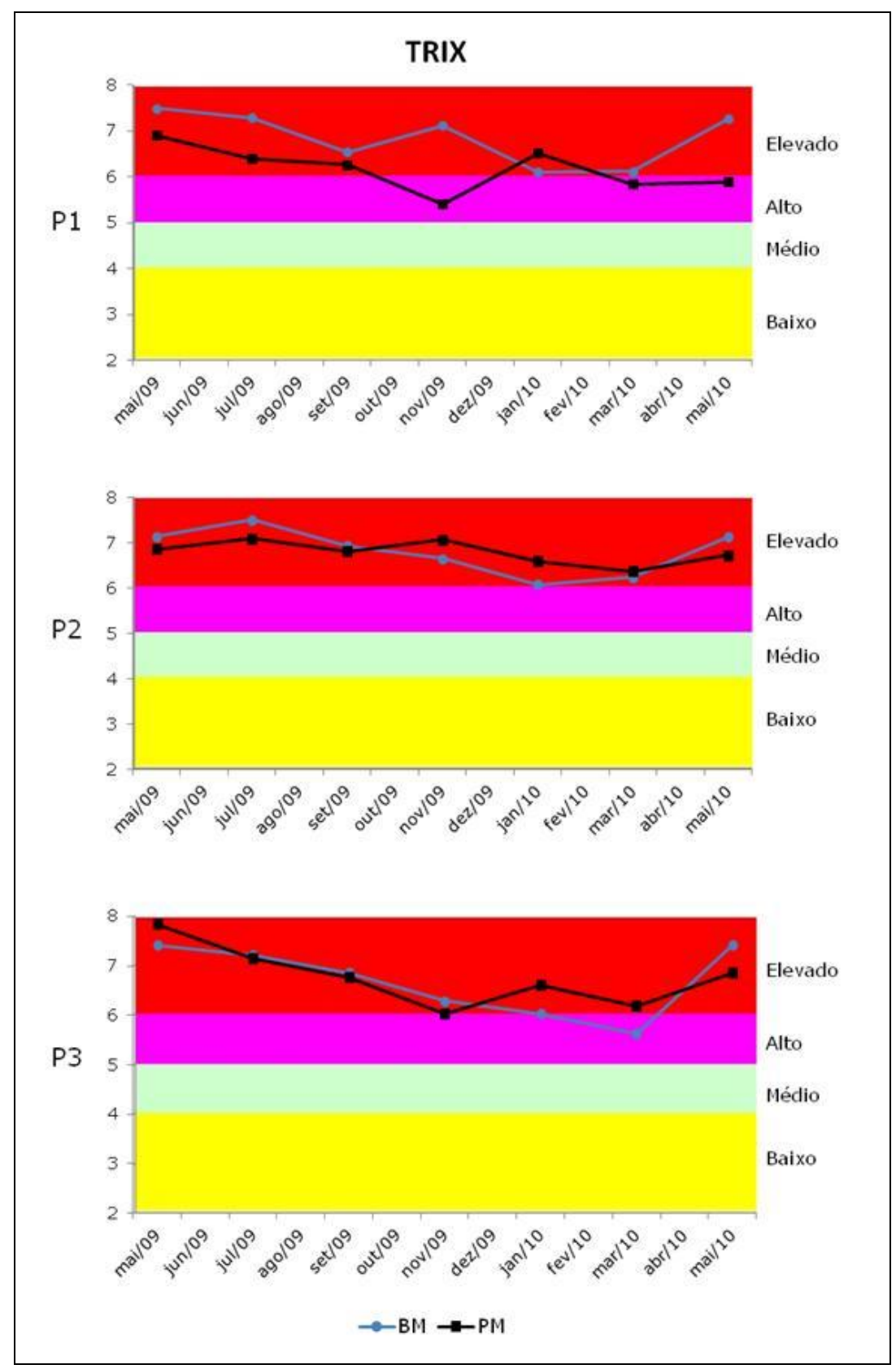

Figura 2 - Variação temporal do TRIX no complexo estuarino em estudo ( $P$ 1: Confluência dos rios Ipojuca e Merepe; P2: rio Merepe e P3: rio Ipojuca), durante a baixa-mar (BM) e preamar (PM).

A distribuição sazonal do TRIX nos estuários dos rios Ipojuca e Merepe, esteve mais elevada no período chuvoso em ambos os estágios de maré (baixa-mar e preamar). Portanto, 
este índice apresenta o período chuvoso como o mais crítico para o sistema, apresentando uma qualidade de água pobre, em decorrência da ação antrópica.

A concentração de clorofila a apresentou os menores valores no P1 durante a preamar, no período chuvoso, com mediana $0,77 \mu \mathrm{g} . \mathrm{L}^{-1}$ e as maiores concentrações foram registradas no mesmo ponto durante a baixa-mar, no período de estiagem, com mediana 8,13 $\mu \mathrm{g}^{-1} \mathrm{~L}^{-1}$. Demonstrando que este parâmetro é fortemente influenciado pelas variações sazonais e de marés.

A taxa de saturação de oxigênio apresentou os menores valores no P3 durante a baixamar, no período de estiagem, com mediana 38,09\% (condições semipoluída) e os maiores valores foram registrados no P1 durante a preamar, no período chuvoso, com mediana $104,45 \%$ (condições supersaturadas). Demonstrando que a maré funciona como um dispersor de gases, sais e nutrientes no ecossistema costeiro, sendo esta responsável pela manutenção da saúde destes ambientes.

O fósforo total apresentou as menores concentrações no P3 durante a baixa-mar,no período seco, com mediana $35,37 \mu \mathrm{g} . \mathrm{L}^{-1}$ e as maiores concentrações foram registradas no mesmo ponto e mesmo estágio de maré, no período chuvoso, com mediana 120,03 $\mu \mathrm{g}^{-1} \mathrm{~L}^{-1}$. Demonstrando que este parâmetro é fortemente influenciado pelas variações sazonais.

Enquanto que o nitrogênio inorgânico total apresentou as menores concentrações no P1 durante a preamar, no período de estiagem, com mediana $218,57 \mu \mathrm{g} . \mathrm{L}^{-1}$ e as maiores concentrações foram registradas no P2 durante a baixa-mar, no período chuvoso, com mediana $535,62 \mu \mathrm{g} \mathrm{L} \mathrm{L}^{-1}$. Observou-se uma tendência destas concentrações diminuírem com a preamar no período seco e aumentarem com a baixa-mar no período chuvoso, demonstrando uma forte influência do material alóctone na área em estudo.

\section{DISCUSSÃo}

Com base na combinação das variáveis utilizadas para a obtenção o índice TRIX, este estudo mostrou que o estado trófico das águas do estuários dos rios Ipojuca e Merepe, variaram de alto a elevado, correspondendo a águas de má qualidade, com o mais altos níveis tróficos encontrados no ponto (P3), localizado no baixo estuário do rio Ipojuca, e os mais baixos no ponto ( $\mathrm{P} 1)$, situado na confluência dos dois estuários e próximo ao mar. De acordo com Caiaffa (1999), Giovanardi e Vollenweider (2004) e Penna et al. (2004), os valores do TRIX variando de 6 a 10 correspondem a condições ambientais bastante degradadas, cujo processo de eutrofização encontra-se em estágios elevados.

Em estudos realizados por Herrera-Silvera (2004), na região costeira do México; por Penna et al. (2004), na costa do Mar Adriático; por Flores Montes et al. (op cit.), em uma área portuária tropical e por Cotovicz Jr. et al. (2011), em um sistema lagunar tropical, foram observados altos valores do TRIX durante os períodos de estiagem, diferentemente dos resultados obtidos no presente estudo, que os valores mais elevados foram observados no período chuvoso, corroborando com Travassos (2011), em seu estudo no estuário do rio Botafogo (PE). Este aumento da quantidade de nutrientes, na estação chuvosa, se deve ao aumento dos aportes pontuais e difusos, carreados por fluxos fluviais mais intensos, e ao escoamento superficial.

Foi observado que as variações sazonais são mais expressivas para o sistema do que as variações de estágios de marés. Uma vez que, para a construção do porto estes estuários tiveram sua foz represada e com isso perderam a comunicação com a baía de Suape e tiveram a conexão com o mar reduzida, esta intervenção alterou toda dinâmica estuarina, afetando diretamente os processos de diluição e dispersão dos nutrientes. De acordo com Tett et al. (2003) a grande contribuição de nutrientes nas regiões costeiras, manifestas com elevadas concentrações de clorofila $a$, se devem a um maior tempo de residência das águas costeiras protegidas, como a bacia do Porto de Recife (FLORES MONTES et al., op cit.), e na área estudada. 
Os valores do TRIX apresentam melhor correlação entre as variáveis clorofila a e percentual de saturação de oxigênio dissolvido corroboram com Moncheva et al. (2001), Giovanardi et al. (2002) e Flores Montes et al. (op cit.). À medida que foram registrados no ponto (P1), durante preamar, no período chuvoso, os valores medianos de menores níveis tróficos, baixas concentrações de clorofila a e maiores taxas de saturação de oxigênio dissolvido.

O TRIX tem se apresentado como uma ferrramenta bastante eficiente no monitoramento da qualidade da água em diferentes locais, entre eles, no Mar Mediterrâneo (AERTEBJERG et al., 2001), no Mar Adriático (GIOVANARDI e VOLLENWEIDER, op cit.), em uma área portuária tropical (FLORES MONTES et al., op cit.), bem como no presente estudo. Onde este índice mostrou eficiência na descrição das características locais e sensibilidade às interferências antrópicas ao meio. Cabe ressaltar que os elevados níveis encontrados através do TRIX nas águas estuarinas, coincidem com elevados níveis tróficos das águas desses rios, encontrados no Monitoramento de Bacias realizado pela Agência Estadual de Meio Ambiente (CPRH, 2012).

\section{CONCLUSÃo}

A partir deste estudo pode-se observar que os estuários dos rios Ipojuca e Merepe estão sob forte pressão antrópica, devido à receptação de elevadas cargas de nutrientes (nitrogênio e fósforo) e as diversas modificações hidrodinâmicas as quais foram submetidos, que provocaram o represamento de suas águas e o aumento do tempo de resiliência. Os impactos dessas atividades resultaram no avanço do processo de eutrofização desses corpos de águas costeiros. Demonstrados através do TRIX, estados tróficos variando de alto a elevado, que atestam a má qualidade destas águas. Deste modo, ressalta-se que a melhoria da qualidade das águas estuarinas só poderá ser conseguida levando-se em consideração as condições socioeconômicas da região e o envolvimento da população e demais atores envolvidos, incluindo melhorias no sistema de monitoramento.

\section{AGRADECIMENTOS}

À Empresa de Petróleo Brasileira (Petrobrás) pelo auxílio financeiro para execução desta pesquisa.

\section{REFERÊNCIAS}

AERTEBJERG, G. et al. Eutrophication in Europea Coastal Waters. European Environmental Agency. Copenhagem, DK. 86p. 2001.

BRAGA, R. A. P.; MOURA, H. F.; DUARTE, M. T. Impactos ambientais sobre a estrutura do manguezal de Suape. In: UNIVERSIDADE FEDERAL DE PERNAMBUCO. Departamento de Biologia Geral. Laboratório de Ecologia. Projeto Avaliação de Impactos Ambientais em

Zonas Estuarinas de Pernambuco. Recife. 32-42p. 1989.

\section{BRICKER, S.B.; FERREIRA, J.G.; SIMAS, T. An integrated methodology for assessment of} estuarine trophic status. Ecological Modeling, 169: 39-60, 2003.

CAIAFFA, E. European marine information system: EUMARIS. Dipartimento Ambiente. Centro Ricerche Casoccia, Roma. Paper presented at the Inter-regional Forum of European Conventions Venice, September 27-28p. 1999

CLOERN, J. E. Our evolving conceptual model of the coastal eutrophication problem. Marine Ecology Progress Series. V. 210: 223-253. 2001.

CONDEPE (Instituto de Desenvolvimento de Pernambuco). Caracterização do complexo estuarino-lagunar da área de Suape (Pernambuco- Brasil) : síntese ecológica. Recife, v. 1.1983

COTOVICZ, L. C. Jr; BRANDINI, N. e KNOPPERS, B. A. Índice Trófico do Sistema Lagunar Mundaú-Manguaba (AL) avaliado através de dois modelos. In: GeoAmb, 2011. Resumos... Rio de Janeiro: Universiddae Federal Fluminense, 2011, 3p. 
CPRH (AGÊNCIA ESTADUAL DE MEIO AMBIENTE). Diagnóstico Socioambiental do litoral sul de Pernambuco. Recife: $C P R H, 2003,87 p$.

CPRH (AGÊNCIA ESTADUAL DE MEIO AMBIENTE). Monitoramento de Bacias Hidrográficas. Recife: $\mathrm{CPRH}, 2012$. Disponível em:

<http://www.cprh.pe.gov.br/monitoramento/bacias_hidrograficas/resultados_monitoramento_b acias/bacia_do_rio_ipojuca/39741\%3B63063\%3B17020510\%3B0\%3B0.asp >. Acesso em: 29 de maio de 2012.

DiRetoria DE hidRografia e nAVEGAÇÃo (DHN). Tábuas das marés. Porto de Suape (Estado de Pernambuco). Disponível em:

http://www.mar.mil.br/dhn/chm/tabuas/30645Set2011.htm Acessado em: 30 março 2012.

FERREIRA, JOÃO GOMES et al. Application and sensitivity testing of a eutrophication assessment method on coastal systems in the United States and European Union. Journal of Environmental Management. 82, 2007, 433-445p.

FLORES MONTES M. de J. et al.The Trophic Status of an Urban Estuarine Complex in Northeast Brazil. Journal of Coastal Research, 64, 2011, 408-411p.

GIOVANARDi, F., CICERO, A. M., FERRARI, C. R., MAGALETTI, E., ROMANO, E.; RiNALDI, A. 2002. Interrelationships between Trophic Index (TRIX) and Productivity Indicators in Italian Marine-Coastal Waters: A Tool for Water Quality Evaluation and Management. In: Brebia C. A. (Ed.). Environment Problems in Coastal Regions IV. Wit-Press, Southampton, Boston. Fourth International Conference on Environmental Problems in Coastal Regions, Rhodes Island, Greece. 380-389.

GIOVANARDI, F.; VOLLENWEIDER, R.A. Trophic conditions of marine coastal waters: experience in applying the Trophic Index TRIX to two areas of the Adriatic and Tyrrhenian seas. Journal of Limnology 63 (2): 199-218, 2004.

GRASSHOFF, F.K., ENRHARDT, M.; KREMLING, K. Methods of seawater analysis. 2 ed. Nova Iorque: Verlag Chemie, 1983. 419 p.

HERRERA-SILVERA, J. A.; COMIN, F.A.; RANDA-CIREROL, N.; TROCCOLI, L.; CAPURRO, L. Coastal water quality assessment in the Yucatan Peninsula: management implications. Ocean \& Coastal Management. 47:625-639p. 2004.

LABORATÓRIO DE METEOROLOGIA DE PERNAMBUCO (LAMEPE), vinculado ao Instituto de Tecnologia de Pernambuco (ITEP). Disponível em:

http://www.itep.br/index.php?option=com_content\&view=article\&id=877\&Itemid $=549$.

Acessado em 30 março 2012.

MONCHEVA, S., GOTSIS-SKRETAS, O., PAGOU, K. AND KRASTEV, A. Phytoplankton Blooms in Black Sea and Mediterranean Coastal Ecosystems Subjected to Anthropogenic Eutrophication: Similarities and Differences. Estuarine, Coastal and Shelf Science, 53: 281-295. 2001.

NEUMANN, V. H. M. L. Geomorfologia e sedimentologia quaternária da área de Suape, Pernambuco (Brasil). Recife, 95 f. Dissertação (Mestrado em Geociências) - Departamento de Gelogia, Centro de Tecnologia, Universidade Federal de Pernambuco. 1991.

NEUMANN, V. H.; MEDEIROS, C.; PARENTE, L.; NEUMANN- LEITÃO, S. KOENING, M. L., Hydrodynamism, sedimentology, geomorphology and plankton changes at Suape area (Pernambuco-Brazil) after a Port Complex Implantation. Anais Academia Brasilleira de Ciências 70(2): 313-323. 1998.

NIMER, E. Pluviometria e recursos hídricos dos estados de Pernambuco e Paraíba. Rio de Janeiro: SUOREN, 1979. 117p.

NIXON, S. W. Coastal marine eutrophication: a definition, social causes, and future concerns. Ophelia, 41, 199-219. 1995. 
PARSONS, T.R.; STRICKLAND, J. D. H. Discussion of spectrophotometric determination of marine plankton pigments with revised equations of as certaining chlorophyll a and carotenoids. Journal of Marine Research, New Haven, v. 21, n. 3, p. 155-163, 1963.

PENNA, N., CAPELLACCI, S. e RICCI, F. The influence of the Po River discharge on phytoplankton bloom dynamics along the coastline of Pesaro (Italy) in the Adriatic Sea. Marine Pollution Bulletin, 48: 321-326. 2004.

SALAS, FUENSANTA; et al. Applicability of the trophic index TRIX in two transitional ecosystems: The Mar Menor lagoon (Spain) and the Mondego estuary (Portugal). Journal of Marine Science, 65: 1442-1448, 2008.

STRICKLAND, J. D. H.; PARSONS, T. R. A pratical handbook of seawater analysis. 2 ed. Ottawa: Fisheries Research Board of Canada, Bulletin n. 167, 1972, 207-211 p.

Tett, P., Gilpin, L. et al. 2003. Eutrophication and some European waters of restricted exchange. Continental Shelf Research, 23: 1635-1671p.

TRAVASSOS, R. K. Análise da qualidade ambiental no estuário do rio Botafogo: determinação do índice trófico e componentes do fósforo na coluna da água. Recife, 2011. 93 f. : Dissertação (mestrado) - Universidade Federal de Pernambuco. CTG.

Oceanografia, 2011.

TUNDISI, Galizia José. Água no século XXI: Enfrentando a Escassez. São Carlos: RiMa , 2003. 248p.

UNESCO. Determination of photosynthetic pigments in sea water. Report of SCOR/UNESCO working group 17 with meat from 4 to 6 june 1964, Paris: monographys on oceanology methodology. p. 69.

UNESCO. 1973. International Oceanographic Tables. Wormly, v. 2,p. 141.

VASCETTA, M., KAUPPILA, P., COMINO, E., ROSSO, M., 2004. Indicators for coastal marine eutrophication: application of Trophic Index TRIX in the northern Gulf of Finland. In: Proceedings of the 14th Meeting of the Italian Society of Ecology. Disponivel em:

<http://www.xivcongresso. societaitalianaecologia.org/articles/>. Acesso em: 10 de abril de 2012.

VOLLENWEIDER, R. A., GIOVANARDI, F., MONTANARI, G.; RINALDI, A. 1998. Characterization of the trophic conditions of marine coastal waters with special reference to the NW Adriatic Sea: Proposal of a trophic scale, turbity and generalized water quality index. Environmetrics, 9:329 $-357$. 